Tôhoku Math. Journ.

36 (1984), 33-40.

\title{
A MODIFIED FORM OF THE VARIATION-OF-CONSTANTS FORMULA FOR EQUATIONS WITH INFINITE DELAY
}

\author{
Dedicated to Professor Tamotsu Tsuchikura on his sixtieth birthday
}

\section{ToshIKI NAITO}

(Received December 22, 1982)

1. Introduction. For equations with finite delay, the variation-ofconstants formula was given in Halanay's book [2]. Banks [1] pointed out a mistake in this book and presented the correct result. Since equations with finite delay were mainly considered, the results were derived under the restrictive hypotheses: the kernel function $\eta(t, \theta)$ of the linear operator $L(t, \cdot)$ (cf. Theorem 2.1) is constant for sufficiently small $\theta<0$.

In the present paper, we start from the following hypotheses: $L(t, \phi)$ is continuous and the phase space for $\phi$ is the general space for equations with infinite delay introduced by Hale and Kato [4]. From the first hypothesis, the Borel measurability of $\eta(t, \theta)$ is naturally induced; from the second, the constant property of $\eta(t, \theta)$ mentioned above cannot be assumed (see Theorem 2.1). The equation related to the fundamental matrix is reduced to the standard equation with infinite delay (Proposition 3.1):

$$
x^{\prime}(t)=L\left(t, x_{t}\right)+h(t),
$$

where $h$ is locally integrable. The representation of solutions in Theorem 3.3, which is already announced in [5], has a form that is somewhat different from the variation-of-constants formula given in [1], [2], [3]. For the special phase space $\mathscr{C}_{r}$ defined in Section 4, our formula is rewritten in a form analogous to the variation-of-constants formula. However, it contains a new term depending on the "exponential limit of the initial function at $-\infty$ ". Finally, we remark that the present result is an extension of the work for autonomous equations [6] to the case of nonautonomous equations.

2. Representation of linear operators. For a function $x:(-\infty, a) \rightarrow$ $C^{n}$, let $x_{t}:(-\infty, 0] \rightarrow C^{n}, t<a$, be defined by $x_{t}(\theta)=x(t+\theta)$ for $\theta$ in $(-\infty, 0]$. Suppose $\mathscr{B}$ is a linear space of functions $\phi, \psi, \cdots$, mapping $(-\infty, 0]$ into $C^{n}$, with a semi-norm $|\phi|,|\psi|, \cdots$ having the following 
properties.

$\left(\mathrm{H}_{1}\right)$ If $x:(-\infty, \sigma+A) \rightarrow C^{n}, A>0$, is continuous on $[\sigma, \sigma+A)$ and $x_{\sigma} \in \mathscr{B}$, then $x_{t}$ also lies in $\mathscr{B}$ and $x_{t}$ is a continuous function of $t$ in $[\sigma, \sigma+A)$.

$\left(\mathrm{H}_{2}\right)$ There exist measurable functions $K(t)$ and $M(t)$ of $t \geqq 0$, nonnegative and locally bounded, such that

$$
\left|x_{t}\right| \leqq K(t-\sigma) \sup \{|x(s)|: \sigma \leqq s \leqq t\}+M(t-\sigma)\left|x_{\sigma}\right|
$$

for $\sigma \leqq t<\sigma+A$ and $x$ having the properties in $\left(H_{1}\right)$.

$\left(\mathrm{H}_{3}\right) \quad|\phi(0)| \leqq K|\phi|$ for $\phi$ in $\mathscr{B}$ and some constant $K$.

Hypothesis $\left(\mathrm{H}_{1}\right)$ implies that the space $\mathscr{B}$ contains the space $\mathscr{C}$ of all continuous functions mapping $(-\infty, 0]$ into $C^{n}$ with compact support. To state the following representation theorem, a definition is needed. A function $f$ mapping $(-\infty, 0]$ into a finite dimensional Banach space, locally of bounded variation on $(-\infty, 0]$, is said to be normalized if $f(0)=0$ and it is continuous to the left in the interior of $(-\infty, 0]$.

TheORem 2.1. Suppose $L: J \times \mathscr{B} \rightarrow C^{n}$, where $J$ is an interval, is a continuous mapping such that $L(t, \phi)$ is linear in $\phi \in \mathscr{B}$ for each $t$ in $J$. Then there exists an $n \times n$ matrix function $\eta(t, \theta)$ for $(t, \theta)$ in $J \times(-\infty, 0]$, locally of bounded variation for $\theta$ in $(-\infty, 0]$, such that

$$
\begin{gathered}
L(t, \phi)=\int_{-\infty}^{0}\left[d_{\theta} \eta(t, \theta)\right] \phi(\theta) \quad \text { for } \quad \phi \in \mathscr{C} \\
\operatorname{Var}(\eta(t, \cdot),[-r, 0]) \leqq c|L(t, \cdot)| K(r) \text { for } \quad r>0,
\end{gathered}
$$

where $c$ is a constant dependent on the norm of $C^{n}$ and the integral in (2.1) is the improper Riemann-Stieltjes integral (cf. [6, Theorem 3.5]). If $\eta(t, \theta)$ in Relation (2.1) is normalized in $\theta$, then it is determined uniquely by $L$ and is Borel measurable for $(t, \theta)$ in $J \times(-\infty, 0]$.

Proof. The first result of the theorem is a direct consequence of Proposition 3.3 and Theorem 3.5 in [6]; Hypothesis $\left(\mathrm{H}_{0}\right)$ in [6] is not used to derive these results. Suppose $\eta(t, \theta)$ is normalized in $\theta$ and Relation (2.1) holds. For $m=1,2, \cdots$, define a function $\chi^{m}(t)$ by the relation $\chi^{m}(t)=-I,-m(t+1 / m) I, 0$ for $t$ in $[0, \infty)(-1 / m, 0),(-\infty,-1 / m]$, respectively, where $I$ is the $n \times n$ identity matrix and 0 the $n \times n$ null matrix. Then by integration by parts one has

$$
\lim _{m \rightarrow \infty} L\left(t, \chi_{-\theta}^{m}\right)=\lim _{m \rightarrow \infty} m \int_{\theta-1 / m}^{\theta} \eta(t, r) d r=\eta(t, \theta) \text { for } \theta<0,
$$

since $\eta(t, \theta)$ is continuous to the left for $\theta<0$. This shows that $\eta(t, \theta)$ is determined uniquely by $L$. Furthermore, it is Borel measurable for 
$(t, \theta)$ in $J \times(-\infty, 0)$ as a limit function of the sequence of continuous functions $L\left(t, \chi_{-\theta}^{m}\right)$. Therefore $\eta(t, \theta)$ is Borel measurable for $(t, \theta)$ in $J \times(-\infty, 0]$ since $\eta(t, 0)=0$ for $t$ in $J$.

q.e.d.

3. Fundamental matrix and representation of solutions. Consider a linear functional differential equation with infinite delay (1.1), where $L: R \times \mathscr{B} \rightarrow C^{n}$ satisfies the assumption in Theorem 2.1 and $h: R \rightarrow C^{n}$ is locally integrable. Since the operator norm $|L(t)|=\sup \{|L(t, \phi)|:|\phi|=1\}$ is a lower semi-continuous function for $t$ in $R$, it is Borel measurable; furthermore, from Lemma 3.1 in [7], $|L(t)|$ is locally bounded for $t$ in $R$. The normalized function $\eta(t, \theta)$, therefore, is locally bounded for $(t, \theta)$ in $R \times(-\infty, 0]$; in fact,

$$
|\eta(t, \theta)| \leqq c|L(t)| K(-\theta) \text { for }(t, \theta) \in R \times(-\infty, 0] .
$$

For the following discussion, we set $\eta(t, \theta)=0$ for $\theta>0$. Following the arguments similar to the proofs of Theorems 2.1 and 2.4 in [4], one sees that for every $(\sigma, \phi)$ in $R \times \mathscr{B}$, there exists a unique solution $x(t, \sigma, \phi, h)$ of Equation (1.1) with $x_{\sigma}=\phi$ which is locally absolutely continuous for $t$ in $[\sigma, \infty)$ and which satisfies Equation (1.1) a.e. for $t$ in $[\sigma, \infty)$.

To introduce the fundamental matrix of Equation (1.1), we consider the equation

$$
\begin{aligned}
x^{\prime}(t) & =\int_{\sigma-t}^{0} d_{\theta} \eta(t, \theta) x_{t}(\theta)+g(t) \quad \sigma \leqq t, \\
x(\sigma) & =a,
\end{aligned}
$$

where $g:[\sigma, \infty) \rightarrow C^{n}$ is locally integrable.

Proposition 3.1. Under the above assumptions for $L$, $\eta$ and $g$, Equation (3.2) is reduced to Equation (1.1) with initial condition $x_{\sigma}=0$. Thus for every a in $C^{n}$ there exists uniquely a locally absolutely continuous function $x(t)$ for $t \geqq \sigma$ such that $x(\sigma)=a$ and that the first relation of (3.2) holds a.e. in $[\sigma, \infty)$.

Proof. Suppose $x(t)$ is a solution having the above properties. If we set $y(t)=0$ for $t<\sigma$ and $y(t)=x(t)-a$ for $t \geqq \sigma$, then $y(t)$ satisfies

$$
y^{\prime}(t)=\int_{\sigma-t}^{0} d_{\theta} \eta(t, \theta) y_{t}(\theta)-\eta(t, \sigma-t) a+g(t)
$$

a.e. in $t \geqq \sigma$. Since $y_{t}$ lies in $\mathscr{C}$ with $\operatorname{supp} y_{t} \subset[\sigma-t, 0]$ for $t \geqq \sigma$, this relation is reduced to the equation $y^{\prime}(t)=L\left(t, y_{t}\right)-\eta(t, \sigma-t) a+g(t)$ a.e. in $t \geqq \sigma$. Since $\eta(t, \theta)$ is Borel measurable and locally bounded for $(t, \theta)$ in $R^{2}$, the function $\eta(t, \sigma-t)$, as a function of $t$, is also Borel measurable and locally bounded on $R$. Hence the equation for $y$ has a 
unique solution such that $y_{\sigma}=0$ : this implies that $x(t)=y(t)+a$ for $t \geqq \sigma$ is a unique solution of Equation (3.2) having the desired properties.

q.e.d.

The fundamental matrix $X(t, \sigma)$ for $(t, \sigma)$ in $R^{2}$ is defined to be the solution of the equation

$$
\begin{aligned}
& \frac{\partial X}{\partial t}(t, \sigma)=\int_{\sigma-t}^{0} d_{\theta} \eta(t, \theta) X(t+\theta, \sigma) \text { a.e. in } t \geqq \sigma, \\
& X(\sigma, \sigma)=I \text { and } X(t, \sigma)=0 \text { for } t<\sigma .
\end{aligned}
$$

In case of finite delay, it is well known that the fundamental matrix has a relation with a certain matrix solution of the formal adjoint equation

$$
y(s)+\int_{s}^{t} y(\alpha) \eta(\alpha, s-\alpha) d \alpha=b \quad s \leqq t,
$$

where $y$ and $b$ are in $\left(C^{n}\right)^{*}$, the space of $n$-dimensional row vectors. In our case, we will see that this relation is also valid.

The following proposition corresponds to Theorem 3.1, Chapter 6, [3] (see also $[1,2]$ ); the difference is that $\eta(t, \cdot)$ now may not be constant on $(-\infty,-r]$ for any $r>0$. Since the proof is omitted in [1], [2], [3], we give it briefly in the manner suggested in [2] along with the estimate for the variation of the solution.

Proposition 3.2. Given $t$ in $R$ and $b$ in $\left(C^{n}\right)^{*}$, Equation (3.3) has a unique solution $y(s)$ for $s$ in $(-\infty, t]$ which is locally of bounded variation. The total variation of $y$ satisfies

$$
\operatorname{Var}(y,[s, t]) \leqq|b|\left\{\exp \left(\int_{s}^{t} c|L(\alpha)| K^{*}(\alpha-s) d \alpha\right)-1\right\}
$$

where $K^{*}(r)=\sup \{K(s): 0 \leqq s \leqq r\}$.

Proof. Suppose $y(s)$ is Borel measurable and locally bounded for $s$ in $(-\infty, t]$ and designate by $(\Omega y)(s)$ the integral term of Equation (3.3). Since $\eta(\alpha, s-\alpha)=0$ for $\alpha \leqq s$, one has

$$
(\Omega y)(s)=\int_{\sigma}^{t} y(\alpha) \eta(\alpha, s-\alpha) d \alpha \quad \text { for } \quad \sigma \leqq s \leqq t,
$$

and $\operatorname{Var}\left(\eta^{\alpha},[\sigma, t]\right)=\operatorname{Var}\left(\eta^{\alpha},[\sigma, \alpha]\right) \leqq c|L(\alpha)| K(\alpha-\sigma)$ for $\sigma \leqq \alpha \leqq t$, where $\eta^{\alpha}(s)=\eta(\alpha, s-\alpha)$. This leads to

$$
\operatorname{Var}(\Omega y,[\sigma, t]) \leqq \int_{\sigma}^{t}|y(\alpha)| c|L(\alpha)| K(\alpha-\sigma) d \alpha,
$$

which implies $\Omega y$ is locally of bounded variation on $(-\infty, t]$. Such a 
function $\Omega y$ is also Borel measurable and locally bounded on $(-\infty, t]$.

From this remark, one can define succesive approximations $y^{m}(s)$ for $m=0,1,2, \cdots$ as $y^{0}(s)=b$ and $y^{m}(s)=b-\left(\Omega y^{m-1}\right)(s)$ for $s \leqq t$. Then, from Inequality (3.1), one has successively

$$
\begin{aligned}
& \left|y^{1}(s)-y^{0}(s)\right| \leqq \int_{s}^{t} c|b||L(\alpha)| K(\alpha-s) d \alpha \\
& \left|y^{2}(s)-y^{1}(s)\right| \leqq \int_{s}^{t} c|L(\alpha)| K(\alpha-s)\left\{\int_{\alpha}^{t} c|b||L(u)| K(u-\alpha) d u\right\} d \alpha,
\end{aligned}
$$

for $s \leqq t$. Since $K(r) \leqq K^{*}(r)$ for $r \geqq 0$ and $K^{*}(r)$ is nondecreasing, one can replace $K(\alpha-s)$ and $K(u-\alpha)$ in the above inequalities by $K^{*}(\alpha-s)$ and $K^{*}(u-s)$, respectively. Thus the following inequality is proved by induction:

$$
\left|y^{m}(s)-y^{m-1}(s)\right| \leqq \frac{|b|}{m !}\left\{\int_{s}^{t} c|L(\alpha)| K^{*}(\alpha-s) d \alpha\right\}^{m} \quad s \leqq t,
$$

for $m=1,2, \cdots$. Therefore $y^{m}(s)$ converges to a function $y(s)$ uniformly on every compact set of $(-\infty, t]$, and

$$
|y(s)| \leqq|b| \exp \left\{\int_{s}^{t} c|L(\alpha)| K^{*}(\alpha-s) d \alpha\right\} \quad s \leqq t .
$$

This implies that $y(s)=\lim _{m \rightarrow \infty} y^{m}(s)=\lim _{m \rightarrow \infty}\left(b-\Omega y^{m-1}(s)\right)=b-\Omega y(s)$, that is, $y(s)$ is the solution of Equation (3.3). Since $y^{m}(s)$ are all Borel measurable for $s \leqq t, y(s)$ is also Borel measurable for $s \leqq t$. Since $\operatorname{Var}(y,[\sigma, t])=\operatorname{Var}(\Omega y,[\sigma, t])$, one obtains (3.4) by using (3.5) and (3.7).

Suppose $z(s)$ is a solution of (3.3) with $b=0$, and set $A_{\sigma}=\sup \{|z(s)|$ : $\sigma \leqq s \leqq t\}$. Then, following arguments similar to the proof of (3.6), one can show that, for $\sigma \leqq s \leqq t$ and $m=1,2, \cdots,|z(s)|$ is not greater than the right hand side of Inequality (3.6) with $|b|$ replaced by $A_{\sigma}$. Therefore $z(s)=0$, in other words, the solution of (3.3) is unique for b. q.e.d.

Let $Y(\sigma, t)$ be the matrix solution of the system

$$
\begin{aligned}
& Y(\sigma, t)+\int_{\sigma}^{t} Y(\alpha, t) \eta(\alpha, \sigma-\alpha) d \alpha=I \text { for } \sigma \leqq t \\
& Y(\sigma, t)=0 \text { for } \sigma>t .
\end{aligned}
$$

From Proposition 3.2, $Y(\sigma, t)$ is locally of bounded variation in $\sigma$. Now, suppose $x(t)$ is the solution of Equation (3.2). By integration by parts, one has

$$
\int_{\sigma}^{t}\left[d_{\alpha} Y(\alpha, t)\right] x(\alpha)+\int_{\sigma}^{t} Y(\alpha, t) d_{\alpha} x(\alpha)=x(t)-Y(\sigma, t) a .
$$

By the same argument as in Theorem 3.2 in Chapter 6 [3], the second 
term on the left hand side becomes

$$
\int_{\sigma}^{t} Y(\alpha, t)\left\{\int_{\sigma}^{t}\left[d_{s} \eta(\alpha, s-\alpha)\right] x(s)\right\} d \alpha+\int_{\sigma}^{t} Y(\alpha, t) g(\alpha) d \alpha .
$$

Using Theorem 2.1, one sees that the Riemann-Stieltjes integral in the first term is the limit of a sequence of Riemann sums which are all Borel measurable for $\alpha$ in $[\sigma, t]$ and whose norms are not greater than $c|L(\alpha)| K(\alpha-\sigma) \sup \{|x(s)|: \sigma \leqq s \leqq t\}$ for $\alpha$ in $[\sigma, t]$. From the bounded convergence theorem, the order of integration and limit operation can be interchanged; thus, one obtains

$$
\begin{aligned}
\int_{\sigma}^{t} Y(\alpha, t) d_{\alpha} x(\alpha)= & \int_{\sigma}^{t}\left[d_{s}\left\{\int_{\sigma}^{t} Y(\alpha, t) \eta(\alpha, s-\alpha) d \alpha\right\}\right] x(s) \\
& +\int_{\sigma}^{t} Y(\alpha, t) g(\alpha) d \alpha, \text { for } \sigma \leqq t
\end{aligned}
$$

Therefore, using the fact that $Y$ satisfies Equation (3.8), one can rewrite Relation (3.9) as

$$
x(t)=Y(\sigma, t) a+\int_{\sigma}^{t} Y(\alpha, t) g(\alpha) d \alpha \quad \sigma \leqq t .
$$

If one takes $a=I$ and $g(t)=0$ for $\sigma \leqq t$, one obtains $X(t, \sigma)=Y(\sigma, t)$ for all $(t, \sigma)$ in $R^{2}$; consequently,

$$
x(t)=X(t, \sigma) a+\int_{\sigma}^{t} X(t, \alpha) g(\alpha) d \alpha \quad \sigma \leqq t .
$$

To demonstrate the main theorem, we introduce linear operators $S(t): \mathscr{B} \rightarrow \mathscr{B}, t \geqq 0$, by $[S(t) \phi](\theta)=\phi(t+\theta)$ for $t+\theta \leqq 0$ and $[S(t) \phi](\theta)=$ $\phi(0)$ for $t+\theta>0$. Hypothesis $\left(\mathrm{H}_{1}\right)$ guarantees that $S(t) \dot{\phi}$ is continuous in $t$ for each fixed $\phi$ in $\mathscr{B}$.

TheOREM 3.3. Suppose $L: R \times \mathscr{B} \rightarrow C^{n}$ is continuous, $L(t, \phi)$ is linear for $\phi$ in $\mathscr{B}$ and $h:[\sigma, \infty) \rightarrow C^{n}$ is locally integrable. Then for every $\phi$ in $\mathscr{B}$ the solution $x(t, \sigma, \phi, h)$ of Equation (1.1) such that $x_{\sigma}=\phi$ is given by

$$
\begin{aligned}
x(t, \sigma, \phi, h)= & \phi(0)+\int_{\sigma}^{t} X(t, s) L(s, S(s-\sigma) \phi) d s \\
& +\int_{\sigma}^{t} X(t, s) h(s) d s \quad \text { for } \quad t \geqq \sigma .
\end{aligned}
$$

Proof. From the superposition principle, it follows that $x(t, \sigma, \phi, h)=$ $x(t, \sigma, \phi, 0)+x(t, \sigma, 0, h)$ for $t \geqq \sigma$. Since $x(t, \sigma, 0, h)$ is a solution of Equation (3.2) with $a=0$ and $g=h$, it is equal to the third term on the right hand side of Relation (3.11). Now consider the function 
$z(t)=x(t, \sigma, \phi, 0)-y(t)$, where, $y(t)=\phi(t-\sigma)$ for $t \leqq \sigma$ and $y(t)=\phi(0)$ for $t>\sigma$. Then $z(t)$ satisfies $z^{\prime}(t)=L\left(t, z_{t}\right)+L\left(t, y_{t}\right)$ a.e. in $t \geqq \sigma$, and $z_{\sigma}=0$. Since $y_{t}=S(t-\sigma) \dot{\phi}$ for $t \geqq \sigma$, from Formula (3.10), it follows that $z(t)$ is equal to the second term on the right hand side of Relation (3.11). Therefore, the relation $x(t, \sigma, \phi, h)=\phi(0)+z(t)+x(t, \sigma, 0, h)$ becomes Relation (3.11) for $t \geqq \sigma$.

q.e.d.

4. Applications to equations with the phase space $\mathscr{C}_{r}$. The representation formula (2.1) is applicable to functions in $\mathscr{C}$. Is it valid for other functions in $\mathscr{B}$ ? A partial information is given in Theorem 4.4 in [6]; for the space $\mathscr{C}_{r}$ defined below, however, a complete answer was obtained in [5]. In this section, we summarize this result with some comments.

For any fixed $\gamma$ in $R$, let $\mathscr{C}_{r}$ be the space of continuous functions $\phi:(-\infty, 0] \rightarrow C^{n}$ such that $\tilde{\phi}(-\infty)=\lim _{\theta \rightarrow-\infty} e^{-r \theta} \phi(\theta)$ exists in $C^{n}$. It is a Banach space with the norm $|\phi|=\sup \left\{e^{-r \theta}|\phi(\theta)|: \theta \leqq 0\right\}$, and it satisfies Hypothesis $\left(\mathrm{H}_{1}, \mathrm{H}_{2}, \mathrm{H}_{3}\right)$. Changing independent variables, one knows that $\mathscr{C}_{r}$ is isomorphic to the space $C\left([-1,0], C^{n}\right)$, the space of continuous functions mapping $[-1,0]$ into $C^{n}$. This observation yields the following result due to Hagemann ([5, Lemma]). If $L: R \times \mathscr{C}_{r} \rightarrow C^{n}$ is continuous and $L(t, \phi)$ is linear for $\phi$ in $\mathscr{C}_{r}$, then there exist matrix functions $\Lambda(t)$ and $\eta(t, \theta)$ such that $\eta(t, \theta)$ is locally of bounded variation for $\theta$ in $(-\infty, 0]$ and that

$$
L(t, \dot{\phi})=\Lambda(t) \tilde{\phi}(-\infty)+\lim _{R \rightarrow \infty} \int_{-R}^{0}\left[d_{\theta} \eta(t, \theta)\right] \dot{\phi}(\theta) \text { for } \phi \in \mathscr{C}_{r} .
$$

If $\eta(t, \theta)$ is normalized in $\theta$, then $\Lambda(t)$ and $\eta(t, \theta)$ are determined uniquely by $L$ and they are Borel measurable.

Let $\omega(\gamma)(\theta), \gamma$ in $R$, be defined as $\omega(\gamma)(\theta)=\exp (\gamma \theta)$ for $\theta \leqq 0$. Then $\mathscr{C}_{\gamma}$ is considered as $\mathscr{C}_{r}=\omega(\gamma) \mathscr{C}_{0}=\left\{\omega(\gamma) \psi: \psi:(-\infty, 0] \rightarrow C^{n}\right.$ is continuous and $\psi(\theta)$ approaches some vector in $C^{n}$ as $\left.\theta \rightarrow-\infty\right\}$. If $\Lambda(t) \neq 0$, then Formula $(2.1)$ is valid only for $\phi$ in $\mathscr{C}_{r}$ with $\tilde{\phi}(-\infty)=0$, which are in the very restricted subclass of $\mathscr{C}_{r}$. On the other hand, if Relation (4.1) holds with $\eta(t, \theta)$ normalized in $\theta$, then Theorem 2.1 says that $\eta(t, \theta)$ is unique for $L$ and is Borel measurable for $(t, \theta)$. If one set $\phi=\omega(\gamma) a$, $a$ in $C^{n}$, then one can compute $\Lambda(t) a$ for every $a$ in $C^{n}$; consequently

$$
\Lambda(t)=L(t, \omega(\gamma) I)-\lim _{R \rightarrow \infty} \int_{-R}^{0} d_{\theta} \eta(t, \theta) e^{\gamma \theta} \text { for } t \text { in } R .
$$

This also shows that $\Lambda(t)$ is unique for $L$ and it is Borel measurable.

Finally, if one applies Representation (4.1) to $L(s, S(s-\sigma) \phi)$ in Formula (3.11), one obtains the following [5, Theorem] 


$$
\begin{aligned}
x(t, \sigma, \phi, h)= & X(t, \sigma) \phi(0)+\left[\int_{\sigma}^{t} X(t, s) \Lambda(s) e^{\gamma(s-\sigma)} d s\right] \tilde{\phi}(-\infty) \\
& +\lim _{R \rightarrow \infty} \int_{-R}^{0} d_{\theta}\left[\int_{\sigma}^{t} X(t, s) \eta(s, \sigma+\theta-s) d s\right] \phi(\theta) \\
& +\int_{\sigma}^{t} X(t, s) h(s) d s \text { for } t \geqq \sigma, \phi \text { in } \mathscr{C}_{r} .
\end{aligned}
$$

This is really an extension of Formula (9) in [1] (see also [3, Theorem 3.2]). In addition to the formal prolongation of the interval of the Riemann-Stieltjes integral, there appears a new term dependent on the limit $\tilde{\phi}(-\infty)$, "the exponential limit of $\phi$ at $-\infty$ ".

\section{REFERENCES}

[1] H. T. BANKs, Representations for solutions of linear functional differential equations, J. Differential Equations 5 (1969), 399-409.

[2] A. Halanay, "Differential Equations: Stability, Oscillations, Time Lags", Academic Press, New York, 1966.

[3] J. K. HALE, "Theory of Functional Differential Equations", Springer-Verlag, New York, Heidelberg, Berlin, 1977.

[4] J. K. HALe AND J. Kato, Phase space for retarded equations with infinite delay, Funkcial. Ekvac. 21 (1978), 11-41.

[5] T. Hagemann and T. NaIto, Functional differential equations with infinite delay on the space $\mathscr{C}_{r}$, Proc. EQUADIFF 82, Lecture Notes in Math. 1017, Springer-Verlag, Berlin, Heidelberg, New York, Tokyo, 1983, 207-214.

[6] T. NAITo, Fundamental matrix of linear autonomous retarded equations with infinite delay, Tôhoku Math. J. 32 (1980), 539-556.

[7] K. Sawano, Exponential asymptotic stability for functional differential equations with infinite retardations, Tôhoku Math. J. 31 (1979), 363-382.

Department of Mathematics

The University of Electro-Communications

ChoFU, Tокуо 182

JAPAN 